Dept of Endocrinology, William Harvey Research Institute School of Medicine and Dentistry

Barts and the London School of Medicine and Dentistry, Queen Mary University of London

\title{
Adrenal pigmentation in PPNAD is a result of melanin deposition and is associated with up-regulation of the melanocortin 1 receptor
}

DM Cavlan, R Carroll, C Evagora, D Berney, H Storr, WM Drake, PJ King

\section{Introduction}

We recently demonstrated that adrenal stimulation with a-MSH alone, independent of ACTH, could result in cortisol production and adrenal pigmentation (See poster P390). We described a case of adrenal pigmentation as a consequence of

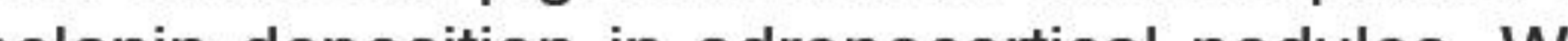
ypothesised that this finding could have mplications for the pathophysiology of othe pigmented adrenal diseases

\section{Primary Pigmented Nodular} Adrenal Disease

Primary pigmented nodular adrenal disease is characterised by small to normal sized adrenal glands containing multiple small pigmented nodules in the cortex ${ }^{1}$. It can lead to an ACTHindependent Cushing's syndrome which usually manifests in childhood and is treated with bilateral adrenalectomy. It may occur in an isolated form or as part of the multiple neoplasia syndrome Carney as part of the multiple neoplasia syndrome Carney
complex (CNC). The co-existing lesions in CNC complex (CNC). The co-existing lesions in CNC pigmented lesions of the skin, as well as adrenocortical and pituitary tumours.

Genetic causes for PPNAD and CNC have been identified in the CAMP-mediated protein kinase A (PKA) signaling pathway. This pathway mediates the adrenal response to ACTH stimulation through he melanocortin 2 receptor (MC2R). Upregulation downstream of receptor binding can therefore lead cortisol excess and Cushing's syndrome. More than $60 \%$ of patients with CNC have mutations in PRKAR1A - one of the regulatory subunits of PKA. Further mutations have been found in the phosphodiesterase enzymes $P D E 11 A$ and $P D E 8 B$ which catalyse the hydrolysis of CAMP and cGMP. Failure to degrade cAMP may therefore lead to an increase in PKA signalling.

The cause of pigmentation in PPNAD has previously been assumed to be a result of pofuscin deposition. This is regarded as a "wea and tear" pigment composed of lipid containing granules of lysosomal digestion. Its presence is a marker of cell aging, or high metabolic activity.

We hypothesised that the pigment in PPNAD was not lipofuscin, but melanin.

\section{Methods}

Institutional ethical review board approval was obtained for this study. Three adult patients who had undergone bilateral adrenalectomy as teenagers for PPNAD consented to the histological examination of their excised adrenal glands. The adrenals from a patient with Cushing's syndrome secondary to an extrapituitary (ectopic) source of ACTH were similarly assessed for comparison.

Serial $3 \mu \mathrm{m}$ sections of adrenal gland were stained using H\&E and the Masson-Fontana method for melanin. Immunohistochemical staining was performed against the following antigens:

11ß-hydroxylase (CYP11B1)

Melanocortin 1 receptor (MC1R)

Human melanoma black-45 (HMB45)

Micro-ophthalmia associated transcription factor (MITF)

1. Horvath A, Stratakis CA. Primary pigmented nodular adrenocortical disease and Cushing's syndrome. Arq Bras

2. Swope VB, Jameson JA, MCFarland KL. Defining MC1R and antagonists agouti signaling protein and $\beta$-defensin $3 . \mathrm{J}$ Invest Dermatol 2012; 132:2255-2262
Results

A brown granular pigment was detected within the nodules of each PPNAD adrenal. It stained black using the Masson-Fontana method, indicating that it was melanin rather than lipofuscin. The zona reticularis (ZR) of the ACTH-stimulated adrenal demonstrated the same pigmentation (Fig 1).

HMB-45 and MITF are markers of the melanosome, the organelle responsible for the generation of melanin. There was positive immunostaining for HMB45 and MITF within the PPNAD nodules and the ZR of the ACTH-stimulated adrenal.

The pigmented nodules in the PPNAD adrenals also demonstrated the strongest staining for CYP11B1 and MC1R. There was widespread ZF and ZR staining in the ACTH-stimulated adrenal.
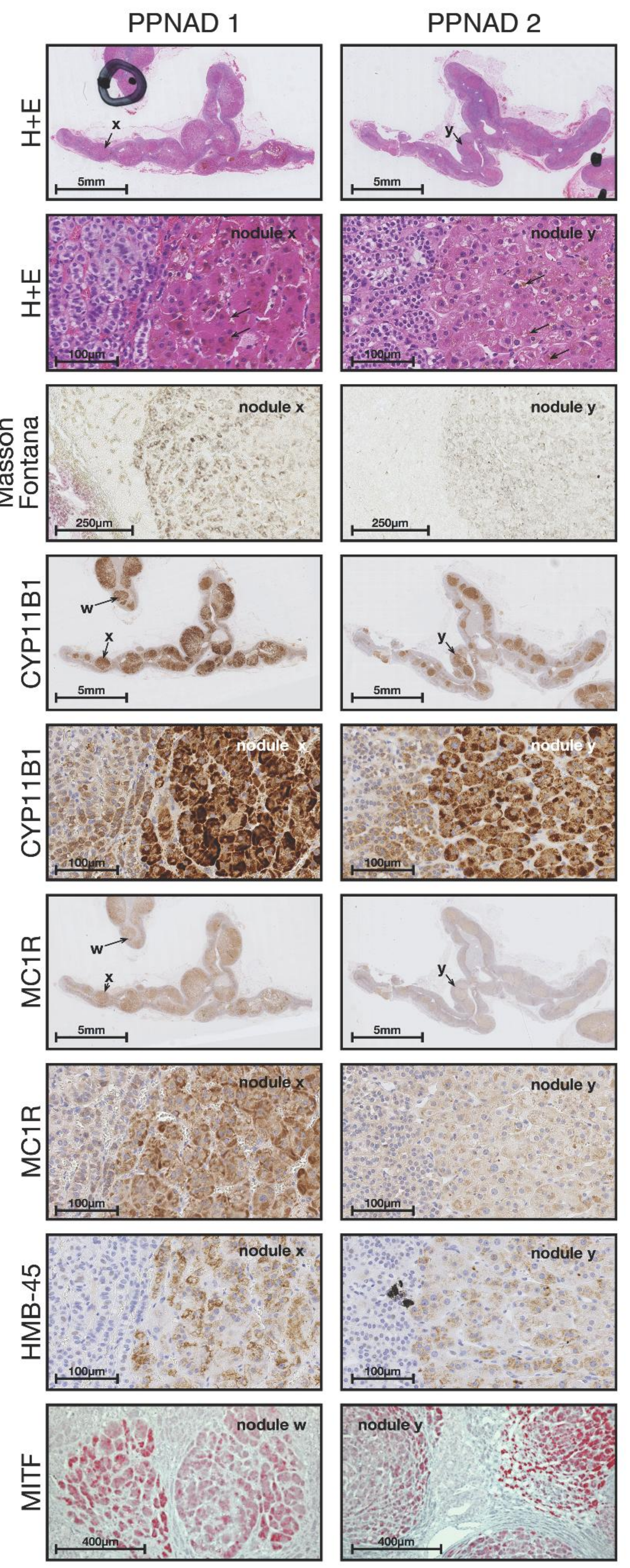

Fig 1. Histochemical and immunohistochemical stain human melanoma black 45 antigen; MITF -

\section{Discussion}

Our previous work demonstrated that a-MSH acting through the MC1R could cause adrenal cortisol production and pigmentation. These histological data suggest that in PPNAD, autonomous up regulation of downstream cAMP signalling pathways can have the same result.

MC1R activation in melanocytes leads to a cAMP-mediated signalling cascade that up-regulates the enzymes associated with melanin production. The precise physiological role of MC1R in normal human adrenal physiology, and the exact mechanism for melanin production has yet to be elucidated. Up-regulation of MC1R in response to chronic activation is well recognised in skin², and the same mechanism may be operating in PPNAD.

The prevalence and implications of ZR pigmentation in the human adrenal gland is an interesting area for future study

PPNAD 3
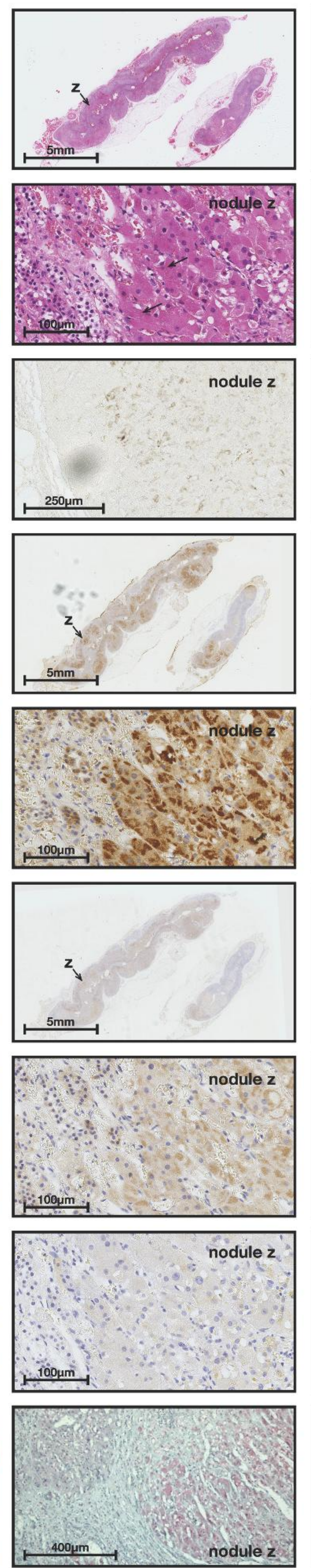

Ectopic ACTH
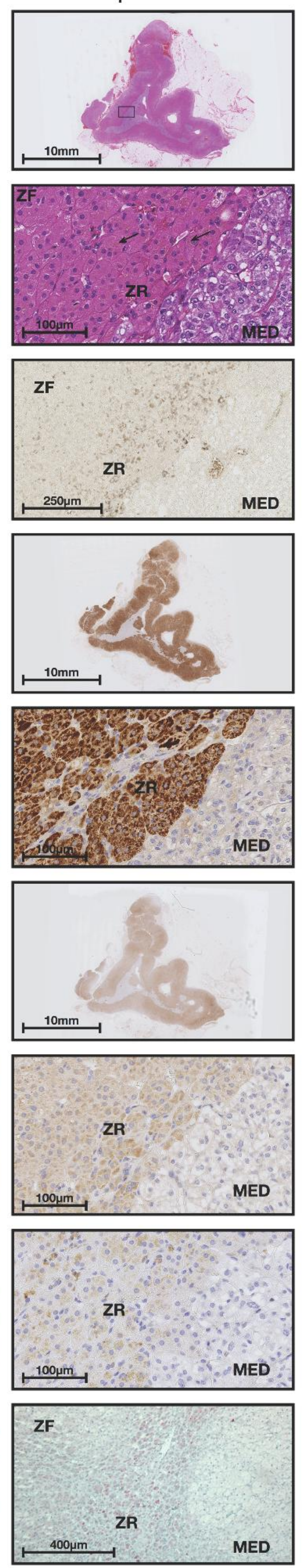
c 